\title{
Quantum models of Parrondo's games
}

\author{
A.P. Flitney*, D. Abbott \\ Centre for Biomedical Engineering (CBME) and Department of Electrical and Electronic Engineering, \\ The University of Adelaide, Adelaide, SA 5005, Australia
}

\begin{abstract}
A Parrondo's paradox is an effect where two losing games, when combined, can produce a net winning result. We provide a short introduction to quantum versions of Parrondo's games and review the current status of the work.
\end{abstract}

(c) 2002 Elsevier Science B.V. All rights reserved.

PACS: 03.67.-a; 02.50.Le

Keywords: Quantum games; Parrondo's games; Quantum lattice gas automata

\section{Introduction}

A Parrondo's paradox results when a game that is losing as a result of some form of feedback becomes winning when the feedback is disrupted by a second losing game that acts as a source of "noise". Classical Parrondo's games have traditionally been cast in the form of gambling games utilizing a set of biased coins. The feedback can take the form of dependence on the gambling capital [1,2], the results of previous games $[3,4]$, or spatial neighbor dependence [5]. For a recent review of Parrondo's games see Ref. [6].

There has been recent interest in exploring the use of quantum field theoretic methods to model classical financial problems [7,8]. Furthermore, inspired by the recent development of quantum game theory [9-11], some theorists have proposed quantum market games [12] where traders can utilize quantum strategies, consisting of superpositions of classical trading actions, to give them an advantage over classical players [13]. There is speculation that the Parrondo effect can be used to produce a profitable

\footnotetext{
* Corresponding author.

E-mail addresses: aflitney@phsyics.adelaide.edu.au (A.P. Flitney), dabbott@eleceng.adelaide.edu.au (D. Abbott).
} 
trading strategy from a combination of individually losing ones [14]. With this in mind we consider quantum versions of Parrondo's paradox.

\section{History dependent games}

In the classical history dependent Parrondo's paradox, game $A$, representing the "noise", is the toss of a single biased coin with winning probability $p=\frac{1}{2}-\varepsilon$, where $\varepsilon$ is a small positive parameter, while game $B$ is a collection of biased coins, the selection of which is dependent on the results of previous games as indicated in Fig. 1. An analysis of game $B$ shows it to be losing for $\varepsilon>0$ when we choose [3]

$$
p_{1}=7 / 10-\varepsilon, \quad p_{2}=p_{3}=1 / 4-\varepsilon, \quad p_{4}=9 / 10-\varepsilon .
$$

However, various sequences of $A$ and $B$, including using a fair coin to select the game to be played at each step, produce a game with a positive expected payoff. The effect can also be obtained by replacing game $A$ with another history dependent game [4].

The history dependent Parrondo's game has been quantized directly by replacing the rotation of a bit, representing a toss of a classical coin, by an $\mathrm{SU}(2)$ operation on a qubit $[15,16]$ :

$$
\hat{A}(\theta, \gamma, \delta)=\left(\begin{array}{cc}
\mathrm{e}^{-\mathrm{i}(\gamma+\delta) / 2} \cos \theta & -\mathrm{e}^{-\mathrm{i}(\gamma-\delta) / 2} \sin \theta \\
\mathrm{e}^{\mathrm{i}(\gamma-\delta) / 2} \sin \theta & \mathrm{e}^{\mathrm{i}(\gamma+\delta) / 2} \cos \theta
\end{array}\right),
$$

where $\theta \in[0, \pi / 2]$ and $\gamma, \delta \in[0,2 \pi]$. Game $B$ acts on the three qubit state $|\psi(t-2)\rangle \otimes$ $|\psi(t-1)\rangle \otimes|\psi(t)\rangle$, where $|\psi(t-1)\rangle$ and $|\psi(t-2)\rangle$ represent the results of the two previous games and $|\psi(t)\rangle$ is the initial state of the target qubit which is modified by the action of $\hat{B}$. The $\hat{B}$ operator consists of four control-control $\mathrm{SU}(2)$ operations, as indicated in Fig. 2.

The initial state $\left|\psi_{i}\right\rangle$ consists of one qubit for each game to be played (equivalent to a pile of coins, each of which is tossed in succession). If $\left|\psi_{i}\right\rangle=|00 \ldots 0\rangle$ the quantum game only gives results that could have been obtained from a classical set up. If we take the initial state to be a superposition, for example, $\left|\psi_{i}\right\rangle=(|00 \ldots 0\rangle+|11 \ldots 1\rangle) / \sqrt{2}$, we produce interference effectively between two different games, those with initial states $|00 \ldots 0\rangle$ and $|11 \ldots 1\rangle$. The payoff (i.e., the count of the number of 1 's in the final

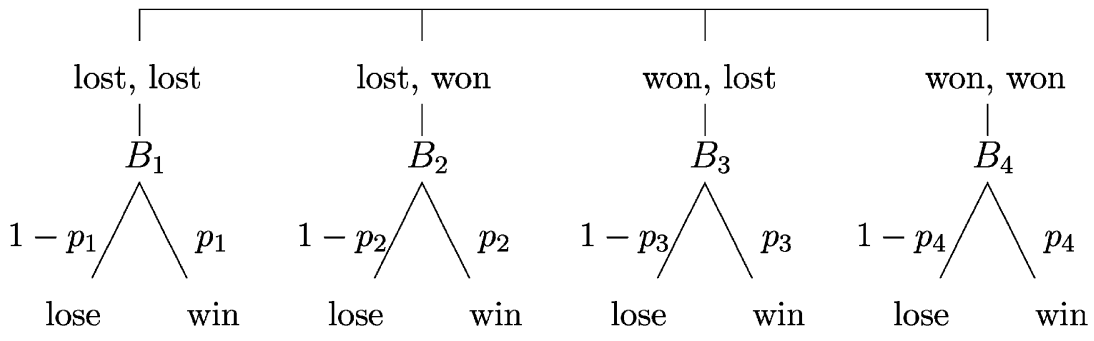

Fig. 1. Winning and losing probabilities for the history dependent game $B$, depending on the last two game results. 


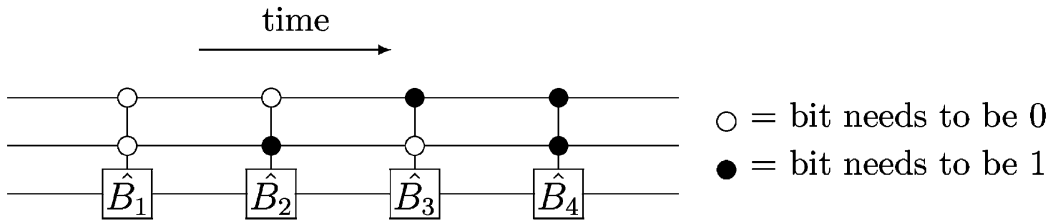

Fig. 2. In the history dependent quantum Parrondo game, $\hat{B}$ consists of four control-control rotations acting on $|\psi(t)\rangle$, depending on the four possible states of the two control qubits, $|\psi(t-2)\rangle$ and $|\psi(t-1)\rangle$. Here, $\hat{B}_{i}=\hat{A}\left(\theta_{i}, \gamma_{i}, \delta_{i}\right)$.

state) is then dependent on the phase angles in the $B_{i}$ operators. By judicious selection of the phases, the extent of the interference can be controlled, either enhancing or diminishing the payoff. For a sample of results see Ref. [16].

\section{Capital or position dependent games}

In capital dependent Parrondo's games, $A$ is again the toss of a single biased coin with winning probability $p=\frac{1}{2}-\varepsilon$, but game $B$ utilizes two coins whose use depends on the total capital of the player: coin $B_{1}$ with winning probability $p_{1}$ is used if the capital is divisible by three, otherwise $B_{2}$ is used with winning probability $p_{2}$. By choosing, for example,

$$
p_{1}=1 / 10-\varepsilon, \quad p_{2}=3 / 4-\varepsilon, \quad \varepsilon>0,
$$

we get a net loss over time [1]. Although the weighted average of the winning probabilities in Eq. (3) is positive for small $\varepsilon$, the "bad" coin $\left(B_{1}\right)$ is used more often than the one-third of the time that we might naively expect. By mixing games $A$ and $B$ this effect is broken and the combination can now be winning provided the net positive effect of game $B$ exceeds the negative effect of game $A$ (see Fig. 3).

In Meyer and Blumer's quantum version of this game [17] the capital corresponds to a discretization of the position of a particle undergoing Brownian motion in one dimension under the action of some potential. A potential with a constant gradient sloping towards negative $x$ produces the effect of game $A$, while game $B$ corresponds to a tilted sawtooth potential. The quantum "coin" is a two state system such as a spin- $\frac{1}{2}$ particle, in a superposition of the $|\uparrow\rangle$ and $|\downarrow\rangle$ states, the eigenstates of $\sigma_{z}$. The quantum analogue of an unbiased coin flip is a unitary transformation represented by the matrix $\frac{1}{\sqrt{2}}\left(\begin{array}{ll}1 & i \\ i & 1\end{array}\right)$. Let $|x\rangle$ correspond to the gambling capital, and $|\uparrow\rangle$ and $|\downarrow\rangle$ indicate a win or a loss, respectively. An unbiased "coin" flip is effected by the unitary transformation

$$
\begin{aligned}
& |x, \downarrow\rangle \rightarrow \frac{1}{\sqrt{2}}(|x-1, \downarrow\rangle+\mathrm{i}|x+1, \uparrow\rangle), \\
& |x, \uparrow\rangle \rightarrow \frac{1}{\sqrt{2}}(\mathrm{i}|x-1, \downarrow\rangle+|x+1, \uparrow\rangle) .
\end{aligned}
$$




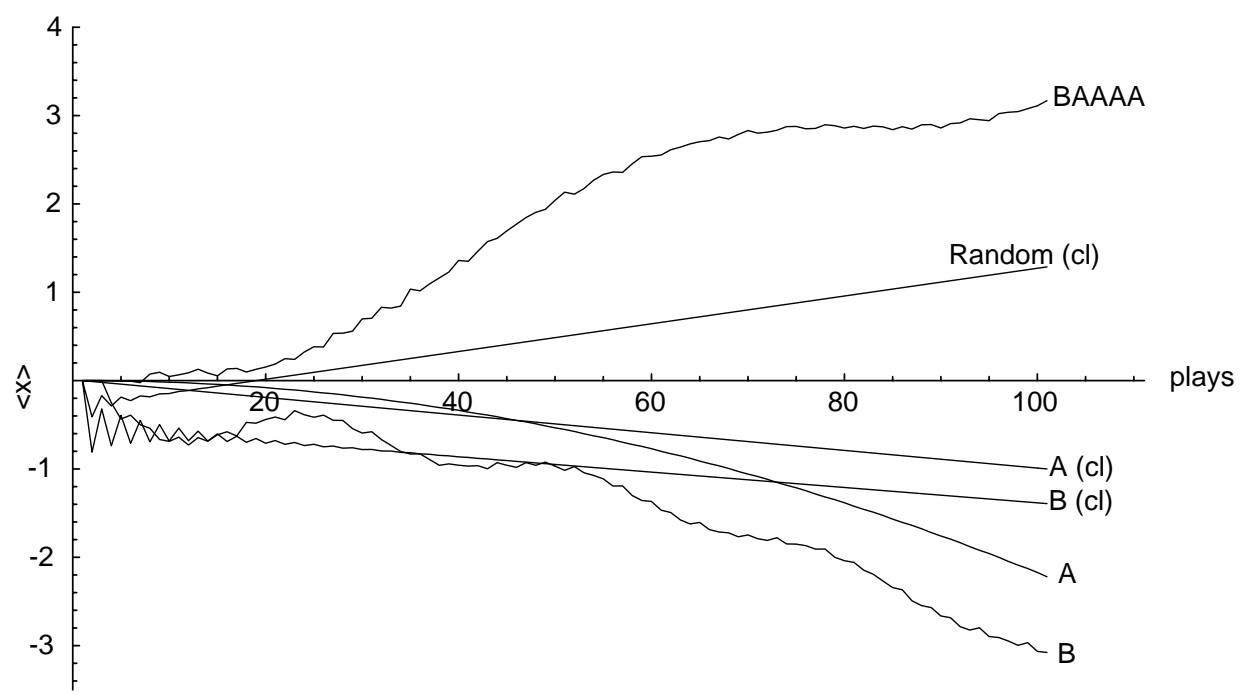

Fig. 3. Comparison of the classical and quantum capital/position dependent Parrondo's games. The classical results (cl) are for $p=\frac{1}{2}-\varepsilon$ for game $A$, and $p_{1}=\frac{1}{10}-\varepsilon$ and $p_{2}=\frac{3}{4}-\varepsilon$ for game $B$, where $\varepsilon=0.005$. "Random" refers to selecting $A$ or $B$ at each play using a fair coin. The quantum results are for $\alpha=2 \pi / 5000$ and $\beta=\pi / 3$.

The initial state is chosen to be $\frac{1}{\sqrt{2}}(|0, \uparrow\rangle+|0, \downarrow\rangle)$ so the particle begins with no particular momentum bias and an unbiased game $A$ produces no net drift. ${ }^{1}$ The effect of the potentials are implemented by multiplication by an $x$-dependent phase factor [18]. The quantum version of the games is the unbiased transition in Eq. (4) multiplied by a phase $\mathrm{e}^{-\mathrm{i} V(x)}$, where for game $A$ and $B, V_{A}(x)=\alpha x$ and $V_{B}(x)=\beta\left(1-\frac{1}{2}(x \bmod 3)\right)+$ $V_{A}(x)$, respectively.

For game $A,\langle x\rangle$ is periodic with period $2 \pi / \alpha$. However, for $\alpha>0,\langle x\rangle \leqslant 0$ for all times, so in this sense game $A$ is losing. The situation is similar for game $B$. Choosing $\alpha=2 \pi / 5000$ and $\beta=\pi / 3$ gives results for the individual games comparable (within a factor of two) to the classical games with the probabilities of Eq. (3). The sequence $B A A A A$ repeating produces one of the greatest positive movements of the particle, as indicated in Fig. 3. For more detail see Ref. [20].

\section{Conclusion}

Classical systems where two losing games can be combined to produce a net winning payoff are known. One of these games can be thought of as noise that disrupts the

\footnotetext{
${ }^{1}$ In Travaglione and Milburn [19] an unbiased quantum random walk is created by using the Hadamard operator, represented by $\frac{1}{\sqrt{2}}\left(\begin{array}{cc}1 & 1 \\ 1 & -1\end{array}\right)$, and starting with the initial state $\frac{1}{\sqrt{2}}(|0, \downarrow\rangle+\mathrm{i}|0, \uparrow\rangle)$. The two schemes are equivalent.
} 
negative bias of the other, thus giving a constructive role to noise. Quantum mechanical analogues of these systems can also produce interesting effects through interference. We have indicated some possible models of quantum Parrondo's games. Using entanglement to combine the games remains to be explored.

\section{Acknowledgements}

This work was supported by GTECH Corporation Australia with the assistance of the SA Lotteries Commission (Australia).

\section{References}

[1] G.P. Harmer, D. Abbott, Stat. Sci. 14 (1999) 206;

G.P. Harmer, D. Abbott, Nature (London) 402 (1999) 864.

[2] G.P. Harmer, D. Abbott, P.G. Taylor, J.M.R. Parrondo, Chaos 11 (2001) 705.

[3] J.M.R. Parrondo, G.P. Harmer, D. Abbott, Phys. Rev. Lett. 85 (2000) 5226.

[4] R.J. Kay, N.F. Johnson, cond-mat/0207386.

[5] R. Toral, Fluct. Noise Lett. 1 (2001) L7.

[6] G.P. Harmer, D. Abbott, Fluct. Noise. Lett. 2 (2002) R71.

[7] K. Illinski, The Physics of Finance: Gauge Modeling in Non-equilibrium Pricing, Wiley, New York, 2001.

[8] B.E. Baaquie, Phys. Rev. E 64 (2001) 016121.

[9] D.A. Meyer, Phys. Rev. Lett. 82 (1999) 1052.

[10] J. Eisert, M. Wilkens, M. Lewenstein, Phys. Rev. Lett. 83 (1999) 3077.

[11] S.C. Benjamin, P.M. Hayden, Phys. Rev. Lett. 87 (2001) 069801;

S.C. Benjamin, P.M. Hayden, Phys. Rev. A 64 (2001) 030301(R).

[12] E.W. Piotrowski, J. Sładkowski, Physica A 312 (2002) 208;

E.W. Piotrowski, J. Sładkowski, Acta Phys. Pol. B 32 (2001) 3873.

[13] E.W. Piotrowski, J. Sładkowski, Physica A quant-ph/0205087, in press.

[14] E. Klarreich, The Sciences 41 (2001) 25.

[15] J. Ng, D. Abbott, in: A. Nowac (Ed.), Annals of the International Society on Dynamic Games, Birkhauser, Boston, in press.

[16] A.P. Flitney, J. Ng, D. Abbott, Physica A 314 (2002) 35.

[17] D.A. Meyer, H. Blumer, J. Stat. Phys. 107 (2002) 225.

[18] D.A. Meyer, Phys. Rev. E 55 (1997) 5261.

[19] B.C. Travaglione, G.J. Milburn, Phys. Rev. A 65 (2002) 032310.

[20] A.P. Flitney, D. Abbott, Proceedings of SPIE Conference on Smart Materials, Nano- and Micro-Smart Systems, Melbourne 16-18th December 2002. 